\title{
Automated Segmentation of the Femur and Pelvis from 3D CT Data of Diseased Hip Using Hierarchical Statistical Shape Model of Joint Structure
}

\author{
Futoshi Yokota ${ }^{1}$, Toshiyuki Okada ${ }^{2}$, Masaki Takao ${ }^{3}$, Nobuhiko Sugano $^{3}$, \\ Yukio Tada ${ }^{1}$, and Yoshinobu Sato ${ }^{3}$ \\ 1 Graduate School of Engineering, Kobe University, Japan \\ 2 Medical Center for Translational Research, Osaka University Hospital, Japan \\ 3 Graduate School of Medicine, Osaka University, Japan
}

\begin{abstract}
Segmentation of the femur and pelvis from 3D data is prerequisite of patient specific planning and simulation for hip surgery. Separation of the femoral head and acetabulum is one of main difficulties in the diseased hip joint due to deformed shapes and extreme narrowness of the joint space. In this paper, we develop a hierarchical multi-object statistical shape model representing joint structure for automated segmentation of the diseased hip from 3D CT images. In order to represent shape variations as well as pose variations of the femur against the pelvis, both shape and pose variations are embedded in a combined pelvis and femur statistical shape model (SSM). Further, the whole combined SSM is divided into individual pelvis and femur SSMs and a partial combined SSM only including the acetabulum and proximal femur. The partial combined SSM maintains the consistency of the two bones by imposing the constraint that the shapes of the overlapped portions of the individual and partial combined SSMs are identical. The experimental results show that segmentation and separation accuracy of the femur and pelvis was improved using the proposed method compared with independent use of the pelvis and femur SSMs.
\end{abstract}

\section{Introduction}

Segmentation of the femur and pelvis from 3D images is an important preprocessing of patient specific planning and simulation for hip surgery. Although healthy hips are relatively easy to be segmented, osteoarthrosis of the hip mainly caused by congenital hip dysplasia needs to be dealt with for clinical application such as preoperative planning for total hip arthroplasty (THA). In the 3D images of patients with the above described disease, bone deformation and joint space narrowing are highly severe, and the boundaries of the femur and pelvis around the joint space are quite difficult to be identified. While 2D X-ray images are typically used for preoperative planning of THA, 3D analysis is quite useful to deal with such highly deformed hips. In the current segmentation methods, 
manual interactions are involved to determine the boundaries around the joint space. Our aim is to automate segmentation of 3D images of deformed hips due to the disease described above.

Automated segmentation of the pelvic bone from 3D images using a statistical shape model (SSM) was reported [1]. For the diseased hip, however, segmentation accuracy is insufficient around the joint space due to lack of boundary information. Some previous works embed spatial relationships between multiple adjacent objects such as the femur and pelvis into SSMs [2] 3]. Frangi et al. constructed a SSM of multiple objects by regarding combined multiple objects as one single shape 2. Yang et al. explicitly modeled the relations between adjacent objects 3]. These methods deal with the relations among whole objects and suitable for stable segmentation of multiple objects due to high specificity of shape representation. In case of the hip joint, the pelvis and femur have close relations locally around the joint space, and thus modeling whole-object relations is inefficient with respect to accuracy of shape representation. To overcome this issue, Okada et al. addressed the problem of embedding local spatial relations into SSMs 44. However, these methods do not deal with intra-patient variability of the spatial relations such as pose variations in joint motion.

In this paper, we propose a method for embedding pose variations of joint motion of the diseased hip into multi-object SSMs and its application to automated segmentation from 3D images. We construct a hierarchical SSM of the hip, in which Frangi's method is firstly applied to construct a combined pelvisfemur SSM of the whole hip, and then Okada's method is utilized to enforce the constraint of local spatial relations around the joint space on individual femur and pelvis SSMs. We aim at stable and accurate segmentation especially around the joint space by applying a coarse-fine strategy using the hierarchical hip SSM.

\section{Methods}

Figure 1 shows coronal views and 3D renderings of CT images of normal and diseased hips. In the normal hip, which is the non-diseased side of a patient data, it is observable in the image that the femoral head and acetabulum are separated by the joint space. In the diseased hip, however, the femoral head is highly deformed and it is difficult to identify the joint space only using local image features. In addition, the shape deformation due to osteoarthrosis of the hip shows a characteristic tendency as the disease progresses. Therefore, anatomical prior information is essential in order to determine the boundaries appropriately.

Anatomical prior information is typically embedded in a SSM. However, the pelvis and femur are interrelated in the hip joint and their spatial relations are changeable under the constraints of the range of motion. Since the pose of the femur against the pelvis is unknown in CT image, not only their shape variations but also the pose variations between them need to be incorporated into statistical modeling. In the following, the detailed methods of the statistical modeling and its use for segmentation are described. 

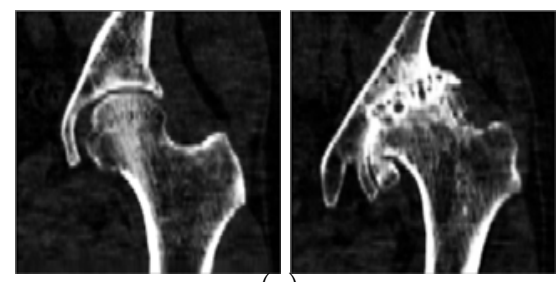

(a)
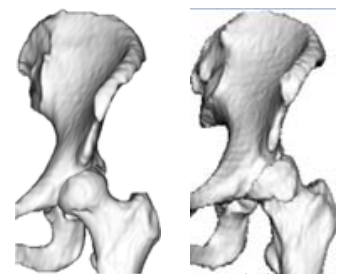

(b)

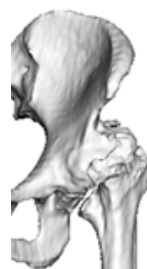

Fig. 1. Coronal views and 3D renderings of CT images of normal and diseased hips. (a) Coronal views. Left: normal hip. Right: diseased hip. (b) 3D renderings. Left: normal hip. Middle: mildly diseased hip. Right: severely diseased hip.

\subsection{Statistical Modeling of Pose and Shape Variations}

We assume that a sufficient number of the surface models of the pelvis and femur of different patients are available, which are constructed from manually segmented regions in the training CT datasets and represented in the pelvis and femur coordinate systems, respectively, defined based on anatomical landmarks. Further, we assume that inter-patient non-rigid registration is performed among the surface models [5].

In order to embed the pose variations into SSMs, possible pose variations are simulationally generated for each patient dataset. Although the hip joint is wellapproximated by a spherical joint if it is healthy, sphere approximation is often inappropriate for the diseased hip joint as shown in Fig. 1(b). We assume that the joint space region, which is defined as a region between the acetabular and femoral boundaries manually traced from CT images, consists of the acetabular and femoral cartilages. Further, we assume that the thickness distribution of the femoral cartilage is uniform while the acetabular cartilage is not. The latter assumption is based on Zoroofi's observation that the acetabular cartilage is mainly damaged in the diseased hip, which was obtained through quantitative MR image analysis [6]. In order to generate possible poses for the diseased case based on the assumptions, we define the distance map of the joint space on the acetabular surface as the distribution of the distance from each point on acetabular surface to the nearest point of the femoral head surface. We regard the distance map in the pose at the CT image acquisition as the template. To generate possible poses of the femur against the pelvis, translation $\mathbf{t}_{j}$ is estimated for each of systematically generated arbitrary rotations, $R_{j}$, so that the calculated distance map is as close as possible to that of the template using the following equation:

$$
\mathbf{t}_{j}=\operatorname{argmin}_{\mathbf{t}} C\left(\mathbf{t} ; \mathrm{R}_{j}\right)=\operatorname{argmin}_{\mathbf{t}} \sum_{k=1}^{N}\left\{d\left(\mathbf{p}_{k}, F\right)-d\left(\mathbf{p}_{k}, \mathrm{R}_{j} F+\mathbf{t}\right)\right\}^{2}
$$

where $d(\mathbf{x}, Y)$ denotes the distance from point $\mathbf{x}$ to surface $Y, \mathbf{p}_{k}$ is each point on the acetabular surface, $F$ is the femoral head surface, and $\mathrm{R}_{\mathrm{j}}$ is each generated 


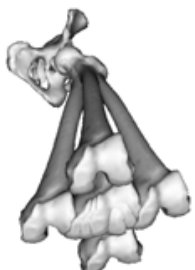

(a)

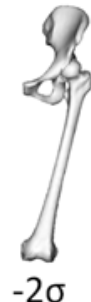

$2 \sigma$

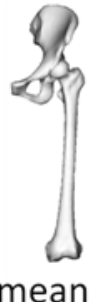

(b)

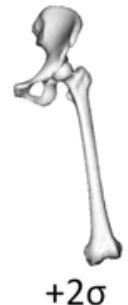

$+2 \sigma$

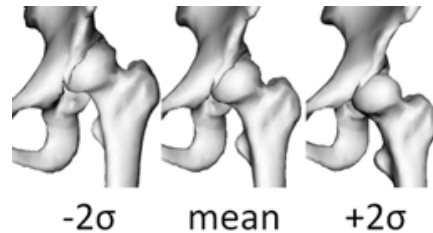

(c)

Fig. 2. Training dataset and two characteristic modes of the constructed combined pelvis and femur SSM. (a) Generated poses of the femur against the pelvis. (b) First mode of SSM. (c) Forth mode of SSM.

rotation matrix. The center of gravity of the femoral head is used for the center of rotation. The femur is rotated along the three axes based on the anatomical coordinate system (e.g., flexion and extension). $C\left(\mathbf{t} ; \mathrm{R}_{j}\right)$ is minimized using Levenberg-Marquardt method.

For each patient data, $n$ poses $\left(\mathbf{t}_{j}, \mathrm{R}_{j}\right)(j=1, \cdots, n)$ of the femur against the pelvis are generated. Figure 2(a) shows examples of poses generated from one patient data. Given $m$ patient data, principal component analysis is applied for $n \times m$ training datasets to construct the combined pelvis and femur SSM where both the shape and pose variations are embedded. Figure 2(b) shows the first mode that represents main variations of both femur pose and pelvis shape. Figure 2(c) shows the fourth mode that mainly represents typical deformation of osteoarthrosis of the hip caused by hip dysplasia.

\subsection{Hierarchical Multi-organ SSM of the Hip Joint}

Specificity and generality are often regarded as criteria of shape representation. The former is related to the ability of representing only a specific category of shapes while the latter is the ability of representing any shapes in the category. The combined SSM is considered to be advantageous on specificity compared with independent pelvis and femur SSMs because it represents only consistent spatial relations of the acetabular and femoral head shapes while inconsistent relations can be accepted especially around the joint space by using the independent SSMs. On the other hand, the independent SSMs are advantageous on generality because more shape variations can be represented by independent use of them.

In order to take the both advantages, a hierarchical multi-organ SSM is constructed, which has the combined SSM at the upper level of hierarchy and the independent SSMs are at the lower level (Fig. 3). In order to embed constraints for maintaining the consistency into the independent SSMs, a partial combined SSM of the acetabulum and proximal femur is constructed using the method described in subsection 2.1 so that pose variations of the acetabulum and proximal femur are embedded. The partial combined SSM is utilized so as to enforce 


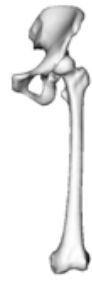

(a)

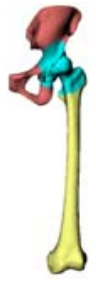

(b)

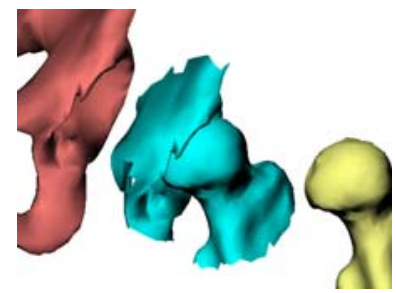

(c)

Fig. 3. Hierarchical division of the hip joint shape for hierarchical statistical shape model. (a) Upper level. (b) Lower level. (c) Partial combined SSM of the acetabulum and proximal femur.

the consistency constraint that the shape variations of independent pelvis and femur SSMs are consistent with those of the partial combined SSM. By enforcing the consistency constraint, it is expected that specificity only around the joint space is improved while generality of the independent SSMs for other parts is still maintained.

Let $\mathbf{s}_{p}$ and $\mathbf{s}_{f}$ be shape parameter vectors in SSMs of the pelvis and femur, respectively. Let $S_{p}\left(\mathbf{s}_{p}\right)$ and $S_{f}\left(\mathbf{s}_{f}\right)$ be polygon surfaces of the pelvis and femur SSMs generated by $\mathbf{s}_{p}$ and $\mathbf{s}_{f}$, respectively. Let $\mathbf{s}_{j s}$ and $S^{\prime}{ }_{j s}\left(\mathbf{s}_{j s}\right)$ be the shape parameter vector and the generated surface of the combined partial SSM, and let $S_{a}^{\prime}\left(\mathbf{s}_{j s}\right)$ and $S_{p f}^{\prime}\left(\mathbf{s}_{j s}\right)$ be the surfaces, which are included in $S_{j s}^{\prime}\left(\mathbf{s}_{j s}\right)$, corresponding to the acetabulum and proximal femur, respectively. Let $S_{a}\left(\mathbf{s}_{p}\right)$ and $S_{p f}\left(\mathbf{s}_{f}\right)$ be the surfaces, included in $S_{p}\left(\mathbf{s}_{p}\right)$ and $S_{f}\left(\mathbf{s}_{f}\right)$, corresponding to $S_{a}^{\prime}\left(\mathbf{s}_{j s}\right)$ and $S_{p f}^{\prime}\left(\mathbf{s}_{j s}\right)$, respectively. The consistency constraints are realized by adding the term $C_{c}\left(\mathbf{s}_{p}, \mathbf{s}_{f}, \mathbf{s}_{j s}\right)$ given by

$$
C_{c}\left(\mathbf{s}_{p}, \mathbf{s}_{f}, \mathbf{s}_{j s}\right)=d\left(S_{a}\left(\mathbf{s}_{p}\right), S_{a}^{\prime}\left(\mathbf{s}_{j s}\right)\right)^{2}+d\left(S_{p f}\left(\mathbf{s}_{f}\right), S_{p f}^{\prime}\left(\mathbf{s}_{j s}\right)\right)^{2}
$$

to the cost function for segmentation, shape recovery, and so on, where $d\left(S_{1}, S_{2}\right)$ denotes average distance between corresponding nodes in polygon surfaces $S_{1}$ and $S_{2}$. Using this term, the femur and pelvis surfaces generated from the independent pelvis and femur SSMs are constrained by consistent joint space surfaces generated using the combined partial acetabulum and proximal femur SSM.

\subsection{Coarse-Fine Segmentation Procedure}

Using the hierarchical SSM, coarse-fine segmentation is performed. Four anatomical landmark points are manually specified in CT images to determine the pelvis coordinate system. After this manual specification, fully-automated segmentation is performed as described below.

Firstly, initialization is performed. The combined SSM is fitted to the boundary edge points of roughly segmented bone regions extracted using simple thresholding of CT images in order to obtain initial parameter setting for subsequent segmentation processes. Let $\mathbf{s}$ be the shape parameter vector of SSM, $E$ be a set of edge points to be fitted to SSM surface defined by $\mathbf{s}$, and $C_{D}(\mathbf{s}, E)$ be a 
cost function defined based on the average distance between the SSM surface generated with $\mathbf{s}$ and edge points $E$. SSM fitting is performed by obtaining $\mathbf{s}$ which minimizes $C_{D}(\mathbf{s}, E)$.

Secondly, segmentation by SSM fitting is performed at the upper level. Edge detection is performed based on intensity profile analysis along perpendicular direction at each surface point of previously estimated SSM, and SSM fitting to the detected edges are repeated for a fixed number of times. And then, the final result of the upper hierarchical level, that is, fitting result to the combined pelvis and femur SSM, is inherited to the lower level as its initial conditions.

Finally, segmentation by SSM fitting is performed at the lower level. Simultaneous fitting of pelvis and femur SSMs is performed under the consistency constraint, which is realized by adding the term described in subsection 2.2 (Equation (2) ) to the cost function, which is given by

$$
C\left(\mathbf{s}_{p}, \mathbf{s}_{f}, \mathbf{s}_{j s}\right)=C_{D}\left(\mathbf{s}_{p}, E_{p}\right)+C_{D}\left(\mathbf{s}_{f}, E_{f}\right)+\lambda C_{c}\left(\mathbf{s}_{p}, \mathbf{s}_{f}, \mathbf{s}_{j s}\right)
$$

where $\lambda$ is a weight parameter for the consistency constraint. Similarly to the previous step, this fitting process is repeated for a fixed number of times.

\section{Results}

We used 22 CT datasets of female patients of osteoarthrosis of the hip caused by hip dysplasia. Any CT datasets did not include hip implants. By utilizing the mirror-transformed contralateral side, 44 hemi-hips were used for construction of SSMs of the hemi-pelvis and femur. Typically, one side was non-diseased, that is, normal, or mildly diseased compared with the other side. Therefore, the datasets included normal hips to some extent.

In addition to the standard pose in CT imaging, six additional poses of the femur were generated. That is, seven poses were used for one hemi-hip dataset. \pm 10 degrees rotations around each of the three axes were generated and appropriate translations were estimated for each rotation using Equation (1). 308 $(=7 \times 44)$ datasets, which involve both shape and pose variations, were used for SSM construction.

In order to construct the combined partial SSM, the acetabulum and proximal femur regions were defined as regions within $50 \mathrm{~mm}$ from the approximated femoral head center in the reference data used for inter-patient nonrigid registration. Using 44 hemi-hips, leave-two-out cross validations were performed to evaluate segmentation accuracy. The reason of using leave-gtwoh-out method was because two hemi-hips of the same patient used for accuracy evaluation should not be used for SSM construction. We tested several parameter values for the weight of the consistency constraint, and $\lambda=0.01$ was selected.

Table 1 shows accuracy evaluation results of one conventional method, that is, the independent pelvis and femur SSMs, and two proposed methods, that is, the combined pelvis and femur SSM, and the hierarchical hip joint SSM. Manually traced boundaries were used for the gold standard, and the average distance between estimated and gold standard boundaries was used for an error measure. 
Table 1. Evaluation results of segmentation accuracy. Averages of 44 datasets of average distance $[\mathrm{mm}]$ are shown in each SSM. The result of the proposed method around the joint space is shown as bold fonts.

\begin{tabular}{|c|c|c|}
\hline Region of evaluation & Whole hip shape & Around joint space \\
\hline \hline Independent pelvis and femur SSMs & $1.26 \mathrm{~mm}$ & $2.26 \mathrm{~mm}$ \\
\hline Combined pelvis and femur SSM & $1.51 \mathrm{~mm}$ & $2.00 \mathrm{~mm}$ \\
\hline Hierarchical SSM & $1.20 \mathrm{~mm}$ & $\underline{\mathbf{1 . 7 8}} \mathbf{m m}$ \\
\hline
\end{tabular}

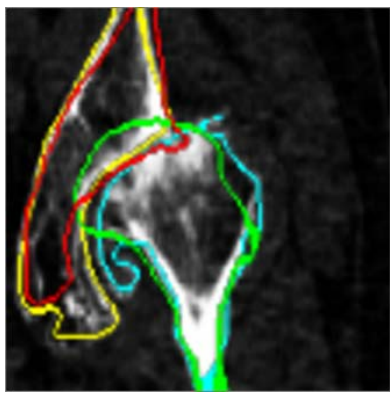

(a)

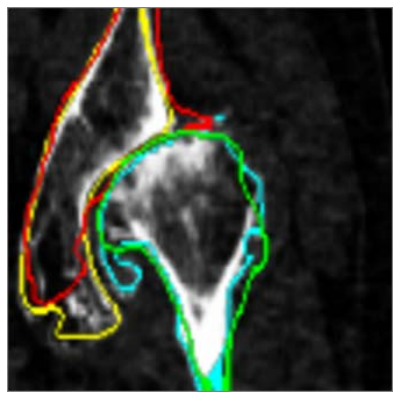

(b)

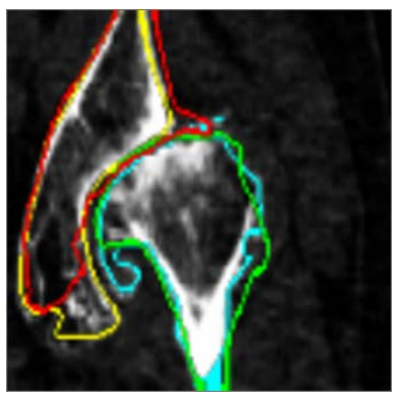

(c)

Fig. 4. Results of an illustrative case. (a) Conventional independent pelvis and femur SSMs. (b) Combined pelvis and femur SSM. (c) Hierarchical SSM. Red and green contours are estimated regions of pelvis and femur, respectively. Yellow and cyan contours are gold standards.

To evaluate the effectiveness of proposed method, we measured the accuracy around the joint space in addition to the whole pelvis and femur shapes. When we used the conventional method, additional manual specification in the CT images was necessary to determine the femur coordinate system. Around the joint space, the error was significantly reduced using the hierarchical SSM (1.78 $\mathrm{mm}$ ) and slightly reduced using the combined SSM $(2.00 \mathrm{~mm})$ compared with the conventional method $(2.26 \mathrm{~mm})$. The accuracy was improved (more than 0.3 $\mathrm{mm}$ ) in 23 cases, worse (more than $0.3 \mathrm{~mm}$ ) in 2 cases, and not changed largely (within $0.3 \mathrm{~mm}$ difference) in 19 cases out of 44 cases by using the hierarchical SSM in spite that additional manual specifications for the femur coordinate system setting was necessary in the conventional method.

Figure 4 shows coronal views of a typical case. Similar to this figure, it was confirmed that the consistency constraint effectively worked to maintain the consistency between the acetabulum and femoral head boundaries in most cases.

\section{Discussion and Conclusions}

We have described a method for modeling statistical prior information of the diseased hip joint structure and its use for automated segmentation from 3D CT 
images. In order to deal with unknown pose between the pelvis and femur, not only shape variations but also pose variations of the two bones are embedded into a SSM to construct the combined pelvis and femur SSM, which is further hierarchically decomposed into the individual pelvis and femur SSMs and a combined acetabulum and proximal femur SSM. The amount of error reduction for the proposed SSMs (21\% reduction) was compareble to that of previously published related method [4 in a similar situation (24\% reduction). We validated that the consistency constraint enforced by the combined acetabular and proximal femur SSM is effective to maintain specific relationships between the acetabulum and femoral head while more accurate shape representation of the whole pelvis and femur becomes possible. The performance of the proposed method depends on the training datasets. Only two cases in which the conventional method significantly outperformed the proposed one in the error measure were those with highly severe deformation (although it is more precise to say that the both methods failed). It is considered that the training datasets did not cover these cases. Excepting few highly severe cases for which training datasets were supposed to be insufficient, however, accuracy improvement was larger for cases with moderate deformation (0.64-mm improvement) than non-diseased cases $(0.49-\mathrm{mm}$ improvement). Therefore, the proposed method was more effective for diseased cases. As future direction, the proposed hierarchical SSM is potentially applicable to other joint structures if the range of motion of the joint can be appropriately modeled.

\section{References}

1. Heiko, S., Dagmar, K., Markus, H., Hans, L., Stefan, Z., Hans, C.H.: Automatic Segmentation of the Pelvic Bones from CT Data Based on a Statistical Shape Model. In: Eurographics Workshop on Visual Computing for Biomedicine, Delft, pp. 93-100 (2008)

2. Frangi, A.F., Rueckert, D., Schnabel, J.A., Niessen, W.J.: Automatic Construction of Multiple-Object Three-Dimensional Statistical Shape Models: Application to Cardiac Modeling. IEEE T. Med. Imaging 21(9), 1151-1166 (2002)

3. Jing, Y., Staib, L.H., Duncan, J.S.: Neighbor-constrained Segmentation with Level Set based 3-D Deformable Models. IEEE T. Med. Imaging 23(8), 940-998 (2004)

4. Okada, T., Yokota, K., Hori, M., Nakamoto, M., Nakamura, H., Sato, Y.: Construction of hierarchical multi-organ statistical atlases and their application to multiorgan segmentation from CT images. In: Metaxas, D., Axel, L., Fichtinger, G., Székely, G. (eds.) MICCAI 2008, Part I. LNCS, vol. 5241, pp. 502-509. Springer, Heidelberg (2008)

5. Haili, C., Anand, R.: A new point matching algorithm for non-rigid registration. Comput. Vis. Image Und. 89, 114-141 (2003)

6. Zoroofi, R.A., Sato, Y., Nishii, T., Nakanishi, K., Tanaka, H., Sugano, N., Yoshikawa, H., Nakamura, H., Tamura, S.: Automated Segmentation of Acetabular Cartilage in MR images of the Hip. Technical Report, Institute of Electronics, Information and Communication Engineers. pp. 63-68 (2005) 